\title{
A!
}

This is an electronic reprint of the original article.

This reprint may differ from the original in pagination and typographic detail.

Wang, L. B.; Saira, O. P.; Pekola, J. P.

\section{Fast thermometry with a proximity Josephson junction}

Published in:

Applied Physics Letters

DOI:

$10.1063 / 1.5010236$

Published: 01/01/2018

Document Version

Publisher's PDF, also known as Version of record

Please cite the original version:

Wang, L. B., Saira, O. P., \& Pekola, J. P. (2018). Fast thermometry with a proximity Josephson junction. Applied Physics Letters, 112(1), 1-5. [013105]. https://doi.org/10.1063/1.5010236

This material is protected by copyright and other intellectual property rights, and duplication or sale of all or part of any of the repository collections is not permitted, except that material may be duplicated by you for your research use or educational purposes in electronic or print form. You must obtain permission for any other use. Electronic or print copies may not be offered, whether for sale or otherwise to anyone who is not an authorised user. 


\section{Fast thermometry with a proximity Josephson junction}

L. B. Wang, O.-P. Saira, and J. P. Pekola

Citation: Appl. Phys. Lett. 112, 013105 (2018);

View online: https://doi.org/10.1063/1.5010236

View Table of Contents: http://aip.scitation.org/toc/apl/112/1

Published by the American Institute of Physics

\section{Articles you may be interested in}

High current density and low emission field of carbon nanotube array microbundle

Applied Physics Letters 112, 013101 (2018); 10.1063/1.4997239

$234 \mathrm{~nm}$ and $246 \mathrm{~nm}$ AIN-Delta-GaN quantum well deep ultraviolet light-emitting diodes

Applied Physics Letters 112, 011101 (2018); 10.1063/1.5007835

Carrier polarity engineering in carbon nanotube field-effect transistors by induced charges in polymer insulator Applied Physics Letters 112, 013501 (2018); 10.1063/1.4994114

Thermoelasticity and anomalies in the pressure dependence of phonon velocities in niobium

Applied Physics Letters 112, 011901 (2018); 10.1063/1.5009617

Cavity enhanced third harmonic generation in graphene

Applied Physics Letters 112, 011102 (2018); 10.1063/1.4999054

Surface recombination velocity imaging of wet-cleaned silicon wafers using quantitative heterodyne lock-in carrierography

Applied Physics Letters 112, 012105 (2018); 10.1063/1.5003260

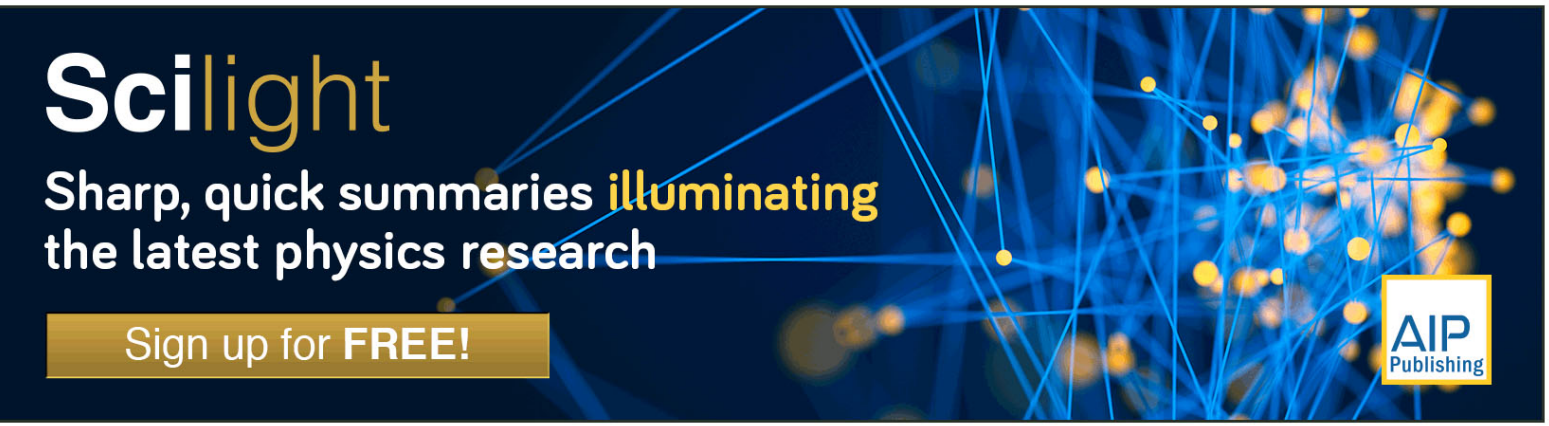




\title{
Fast thermometry with a proximity Josephson junction
}

\author{
L. B. Wang, ${ }^{1, a)}$ O.-P. Saira, ${ }^{2}$ and J. P. Pekola ${ }^{1}$ \\ ${ }^{1}$ Low Temperature Laboratory, Department of Applied Physics, Aalto University, P.O.Box 13500, \\ FI-00076 Aalto, Finland \\ ${ }^{2}$ Department of Applied Physics, California Institute of Technology, 1200 East California Boulevard, \\ MC149-33, Pasadena, California 91125, USA
}

(Received 24 October 2017; accepted 19 December 2017; published online 3 January 2018)

\begin{abstract}
We couple a proximity Josephson junction to a Joule-heated normal metal film and measure its electron temperature under steady state and nonequilibrium conditions. With a timed sequence of heating and temperature probing pulses, we are able to monitor its electron temperature in nonequilibrium with effectively zero back-action from the temperature measurement in the form of additional dissipation or thermal conductance. The experiments demonstrate the possibility of using a fast proximity Josephson junction thermometer for studying thermal transport in mesoscopic systems and for calorimetry. Published by AIP Publishing. https://doi.org/10.1063/1.5010236
\end{abstract}

Thermometry is a cornerstone in studies of thermodynamics. When the investigated system is in equilibrium, the working speed of a thermometer may not be an important factor, as the system status does not change with time. In the past few decades, much progress has been made in understanding thermal transport in nanoscale systems in the steady state. ${ }^{1-3}$ If the timescale of interest is shorter than thermal relaxation time $\tau$ of the relevant system, one needs to measure the system temperature with a fast thermometer in nonequilibrium. The relaxation time increases with lowering temperature, which makes the thermal relaxation time of electrons experimentally accessible at millikelvin temperatures. A thermometer with a large bandwidth is needed to expand the temperature range and the variety of processes that can be observed in non-equilibrium.

Fast thermometry with a sub- $\mu$ s time resolution has been realized with Normal Metal-Insulator-Superconductor (NIS) tunnel junctions and superconducting weak links embedded in resonant circuits. ${ }^{4-8}$ In these methods, the measurement bandwidth is set by the linewidth of the resonant circuit, which cannot be increased indefinitely without sacrificing the readout sensitivity. Recently, Ref. 9 has shown nanosecond thermometry using a superconducting nanobridge, introducing the hysteretic JJ as a fast thermometer for calorimetry with easy integration.

In this letter, we perform fast, minimally invasive thermometry of an evaporated thin-film using proximity JJs. Instead of using a superconducting nanobridge, we utilize proximity JJs consisting of a normal metal weak link contacting two superconducting leads. ${ }^{10,11}$ The normal section of the weak link is galvanically connected to the thin film under study, whose electron temperature can be elevated by Joule heating pulses. We devise a probing scheme that allows us to study the nonequilibrium electron temperature in the thin film with a $\mu$ s time resolution, vanishing dissipation, and virtually zero added heat conductance to the system under study prior to the measurement pulse. Experimental results show the great potential of using a proximity JJ

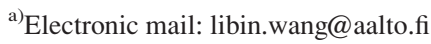

thermometer for precision and fast measurements of electron temperature in metallic films.

The JJ thermometer consists of a normal metal wire (pink) sandwiched between $\mathrm{Al}$ superconducting electrodes (blue), shown in the Scanning Electron Microscopy (SEM) image in Fig. 1(b). The inner four electrodes are used in the experiment to measure the switching current of the JJ, and the other two $\mathrm{Al}$ electrodes at the end of the wire are used to determine the contact resistance between $\mathrm{Al}$ and normal metal. The thermometer is connected to interdigital normal metal films by a narrow metal wire. In Fig. 1(a), we show the whole device structure together with the measurement circuit. Two interdigital normal metal films (pink and cyan) form thermal coupling to the local substrate without a galvanic connection. Electrons in one of the films (cyan) are Joule heated by applying current through $\mathrm{Al}$ electrodes to the normal metal heater. Currents with different polarities $\left(I_{H}^{+}, I_{H}^{-}\right)$are applied to the heater contacts to ensure no heating current flows through the weak link. The length of the

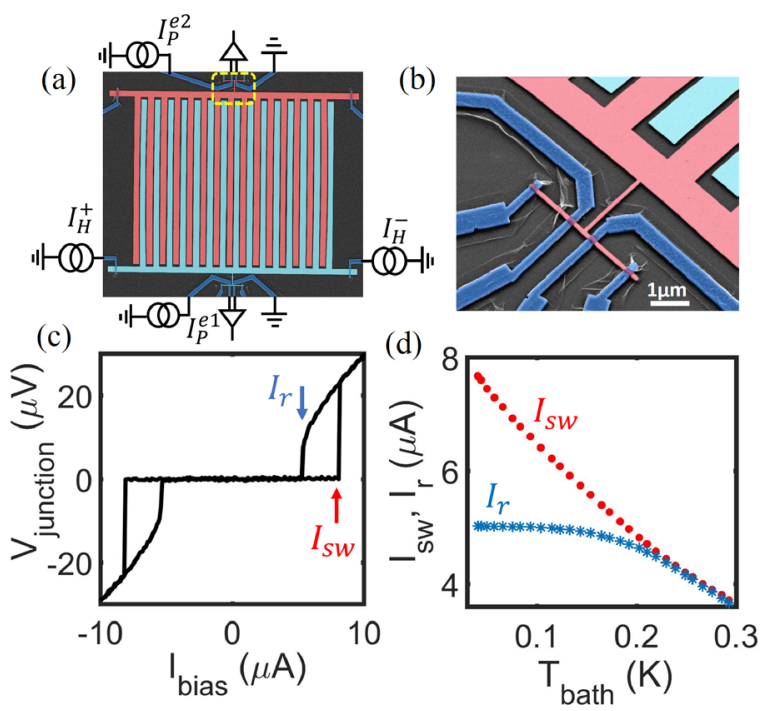

FIG. 1. (a) False-color SEM images of a device and its measurement circuit: $\mathrm{Ag}$ (cyan and pink) and Al (blue); scalebar $4 \mu \mathrm{m}$. (b) Zoom of the dashed yellow area in (a) shows the JJ thermometer. (c) I-V curve of the thermometer shows hysteresis at $60 \mathrm{mK}$. (d) Temperature dependence of $I_{s w}$ and $I_{r}$. 
normal metal heater is $42 \mu \mathrm{m}$ with the resistance of $8.5 \Omega$. The calculated electron diffusion time $\tau_{D}=\frac{L^{2}}{D}$ in the heater is around $40 \mathrm{~ns}$, where $L$ is the junction length and $D$ $=140 \mathrm{~cm}^{2} / \mathrm{s}$ is the diffusion constant. Typically, for metals at low temperature, the electron-electron relaxation time is around $1 \mathrm{~ns}$, which is much smaller than the diffusion time in the metal film, indicating a well-defined Fermi distribution in the films. Within the temperature range studied in this experiment, the wavelength of the thermal phonons in metal films and in the substrate is typically on the order of micrometres, and then, the two metal films with a distance of $200 \mathrm{~nm}$ from each other have the same phonon temperature as the local substrate.

The devices are fabricated on a silicon wafer coated with $300 \mathrm{~nm}$ silicon dioxide. Two-step e-beam lithography is used to define normal metal films and superconducting electrodes separately. Metal films with a thickness of $50 \mathrm{~nm}$ are first deposited by e-beam evaporation. Before contacting normal metal with $\mathrm{Al}$, argon plasma cleaning is used to remove the residual resist from the surface of the normal metal, followed by deposition of $3 \mathrm{~nm}$ of Ti between $\mathrm{Al}$ and the metal film to ensure a good contact. Devices are cooled down using a homemade plastic dilution refrigerator. The sample stage is covered by two shields to screen the electromagnetic radiation to the sample. All measurement lines are filtered with on-chip RC filters at the temperature of the mixing chamber of the refrigerator.

The switching process of a JJ is known to exhibit stochastic character due to thermal and quantum fluctuations. ${ }^{12}$ In the case of DC measurements, by ramping up biasing current through JJ, one can drive the JJ from the superconducting state to the resistive state as shown in Fig. 1(c). Here, the junction shows an $I_{s w}$ value of $7.8 \mu \mathrm{A}$ with a normal state resistance of $3 \Omega$, and the switching current $I_{s w}$ is defined as the corresponding biasing current when JJ switches to the resistive state. When sweeping back the biasing current, the junction switches from the resistive state to the superconducting state at a biasing current well below $I_{s w}$. This suppression of the retrapping current $\left(I_{r}\right)$ originates from the overheating of the electrons in metal wires after the junction switches to the resistive state. For the measured device, thermal hysteresis is observed at temperatures up to $250 \mathrm{mK}$. Importantly, the dissipation begins only after the switch to the normal state. Hence, the statistics of the switching current provide information about the unperturbed film temperature. For all the junctions measured, the calculated Thouless energy $\epsilon_{c}$ is about $30 \mu \mathrm{eV}$, much smaller than the superconductor gap of $\mathrm{Al}(\Delta \approx 200 \mu \mathrm{eV})$, indicating that the system is in the long junction limit. ${ }^{13}$ Temperature calibration of the JJ thermometer is obtained by varying the bath temperature of the refrigerator and recording the $I_{s w}$, as shown in Fig. 1(d). $I_{s w}$ depends almost linearly on temperature without saturation down to $60 \mathrm{mK}$. With this calibration, the SNS JJ serves as an electron thermometer.

In the steady state, considering a system with constant heating $\dot{Q}_{H}$ applied to it, the change in system temperature can be expressed as

$$
\Delta T_{e}=\frac{\dot{Q}_{H}}{G_{t h}}
$$

Here, $\Delta T_{e}=T_{e}-T_{p}$, and $G_{t h}$ is the thermal conductance from the system to its environment. For normal metal, it is well known that electrons (e) are decoupled from the phonon (p) environment at low temperatures and thermal conductance between electrons and phonons $\left(G_{e-p}\right)$ is the bottleneck for energy dissipation at low temperature, ${ }^{14-16}$ which leads to the hot electron effect when $G_{e-p} \ll G_{K}$. Here, $G_{K}$ characterises the phonon-mediated heat transport from the metal lattice to the substrate. Theories and experiments show that the energy flow rate $P_{e-p}$ from the electron gas at temperature $T_{e}$ to the phonon gas at $T_{p}$ is $P_{e-p}$ $=\Sigma V\left(T_{e}^{n}-T_{p}^{n}\right)$. Here, $V$ is the metal volume, and the exponent $n$ and the material specific e-p coupling constant $\Sigma$ will be discussed later in detail. The thermal conductance between electrons and phonons is $G_{e-p}=n \Sigma V T_{e}^{n-1}$ for small temperature differences, i.e., when $T_{e} \approx T_{p}$.

We utilize the hot electron effect under steady state conditions to measure the e-p coupling constant in normal metal. Electron temperature in the metal film is elevated by applying constant heat $\dot{Q}_{H}$ on it while measuring its electron temperature $\left(T_{e 1}\right)$ and also the electron temperature of the indirectly heated metal film $\left(T_{e 2}\right)$. Figure 2(b) shows the heat flow in the system. Joule heating $\left(\dot{Q}_{H}\right)$ applied to the metal film heats up its electrons, and the electrons are coupled to phonons with energy flow rate $\dot{Q}_{e-p}$. Acoustic mismatch theory suggests that the Kapitza resistance between solids can be approximated by $R_{K} A T^{3} \sim 20 \mathrm{~K}^{4} \mathrm{~cm}^{2} / \mathrm{W}, A$ is the contact surface area. At $300 \mathrm{mK}$, the calculated Kapitza resistance is on the order of $10^{6} \mathrm{~K} / \mathrm{W}$, which is negligible compared to the measured thermal resistance between electrons and phonons at the same temperature. The difference in the magnitude will be further pronounced at lower temperatures. The lattice phonon temperature of the two films $\left(T_{p}\right)$ is kept constant at substrate phonon temperature $\left(T_{\text {sub }}\right)$. Meanwhile, $T_{e 2}=T_{p}$ as there is no energy flow between electrons and phonons in the steady state in the indirectly heated film. The substrate temperature $\left(T_{\text {sub }}\right)$ near metal films may
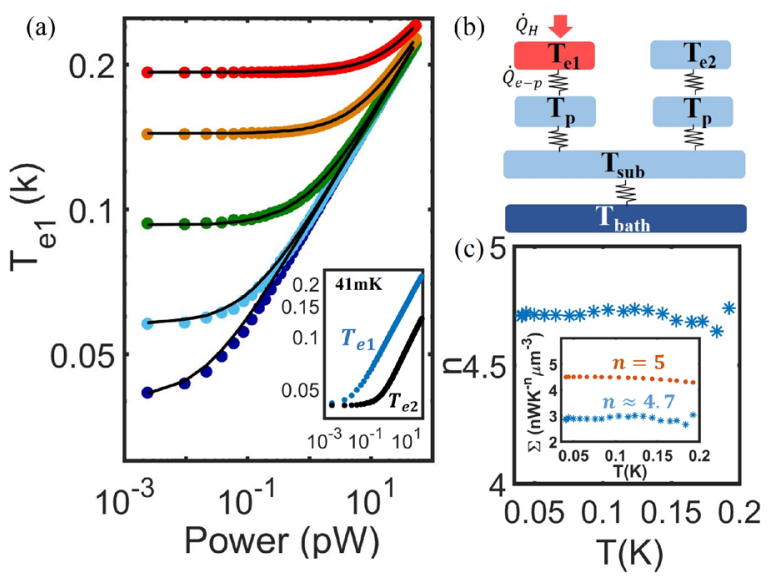

FIG. 2. (a) Electron temperature of the heated film as a function of the heating power applied. Black lines are fits with Eq. (2) with $T_{\text {bath }}=41,57,93,144$, and $192 \mathrm{mK}$ from blue to red. Inset: $T_{e 1}$ and $T_{e 2}$ against heating power at a bath temperature of $41 \mathrm{mK}$. Units are the same as in the main plots. (b) The thermal model of heat flows in the system. (c) Temperature dependence of exponent $n$ obtained by fitting of measurements to Eq. (2) with $n$ and $\Sigma$ being free parameters. Inset: $\Sigma$ as a function of temperature of an $\mathrm{Ag}$ film with a thickness of $50 \mathrm{~nm}$, fitted by fixing $n$ to 5 (yellow stars) and 4.7 (blue dots). 
show a higher temperature than the bath temperature of the refrigerator as Joule heating applied on the metal film heats up the local substrate phonons as well. Electron temperature of the heated $\mathrm{Ag}$ film as a function of the heating power applied is shown in Fig. 2(a). In the inset of Fig. 2(a), we show the increased substrate temperature with respect to the bath temperature of the refrigerator at the bath temperature of $41 \mathrm{mK}$.

With temperature below about $300 \mathrm{mK}$, heat flow through $\mathrm{Al}$ contacts is negligible because of the good thermal isolation of superconducting $\mathrm{Al} .{ }^{17}$ Then, in the steady state, the dominant mechanism for electrons in the heated film to cool is e-p scattering. So, in steady states, we have

$$
I^{2} R=\Sigma V\left(T_{e 1}^{n}-T_{e 2}^{n}\right)
$$

Fitting measurement results shown in Fig. 2(a) to Eq. (2) with $\Sigma$ and $n$ being free parameters at each bath temperature point, we get $n \approx 4.7$ for the Ag film between 40 and $200 \mathrm{mK}$, as shown in Fig. 2(c).

The exponent $n$ in Eq. (2) was first measured by Roukes et al. ${ }^{18}$ for pure $\mathrm{Cu}$ films, and experiments show that the exponent $n$ equals 5, which can be explained by a theory based on a clean three-dimensional free-electron model with $q l \gg 1$. Here, $q$ is the phonon wave factor, and $l$ is the electron mean free path. Phonon wave factor $q$ can be further expressed as $q=h v_{S} / k_{B} T$, where $v_{S}$ is the speed of sound of the phonon mode in metal, $k_{B}$ is the Boltzmann constant, and $h$ is the Planck constant. In a dirty limit with $q l<1$, theories predict that $n$ ranges from 4 to 6 depending on the type and level of disorder. ${ }^{19,20}$ Deviations from $n=5$ are experimentally observed in normal metals and alloys. ${ }^{21-24}$ But this hypothesis is still not fully verified as some experiments show that even for samples in the dirty limit, the energy flow rate from electrons to phonons follows the $T^{5}$ dependence. $^{25,26}$ The effects of phonon dimensionality and substrate properties on e-p coupling have also been discussed..$^{21,27-29}$ Experiments show that $n$ falls below 4.5 for phonons in two dimensions. ${ }^{27}$ Although there are some discussions about the exponent, $n=5$ is still the mostly observed dependence in metal films, and it is widely used in modelling e-p coupling problems. In our experiments, the low temperature resistivity of the films is $1 \times 10^{-8}$ $\Omega \mathrm{m}$, which gives a mean free path of $81.5 \mathrm{~nm}$. In the temperature range of $60-200 \mathrm{mK}, q l \approx 0.2-1.3$.

By fitting measurement results to Eq. (2) with a fixed exponent $n=5$, we get the temperature dependence of the e-p coupling constant $\Sigma$ of Ag shown in the inset of Fig. 2(c). In the same plot, we also show the results by fitting experimental data with $n \approx 4.7$. $\Sigma$ shows essentially no temperature dependence as expected with a constant value of about $4.5 \mathrm{nW} \mathrm{K}^{-5} \mu \mathrm{m}^{-3}$, compatible with earlier measurements on Ag films, ${ }^{30}$ proving the accuracy of our JJ thermometer.

The switching process of JJ can also be probed by sending a current pulse to the junction and measuring its response. The probability of the junction to switch to the normal state depends on the amplitude and the width of the current pulse sent to the JJ due to the stochastic character of the switching process. ${ }^{31}$ During the measurement, a series $(N=50)$ of rectangular current pulses, shown in Fig. 3(a), are sent to the junction and its response is recorded. Each

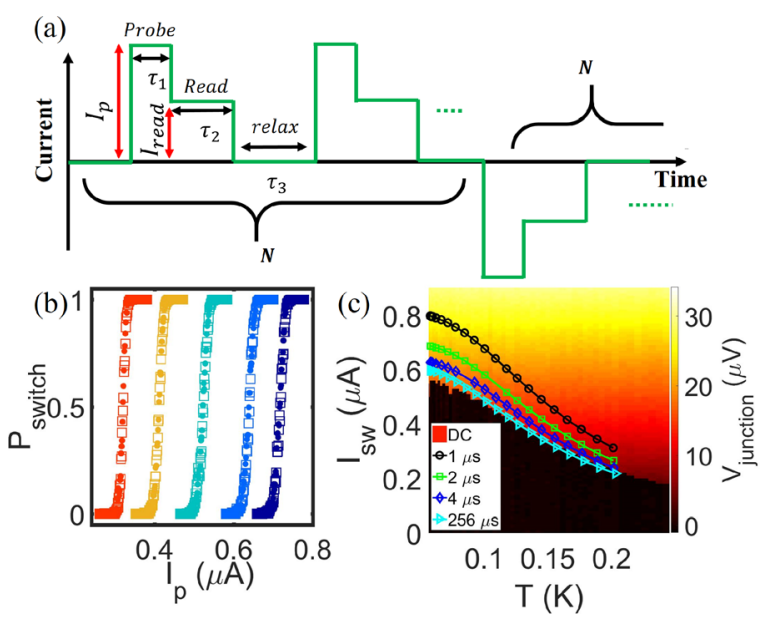

FIG. 3. (a) The waveform of the current pulse train used in determining $I_{s w}$. (b) Switching probability as a function of probe pulse amplitude at different bath temperatures. $T_{\text {bath }}=60,90,121,151$, and $182 \mathrm{mK}$ from blue to red. (c) Temperature dependence of $I_{s w}$ with varying probe pulse widths. DC measurement results are shown in the colour plot.

current pulse consists of two parts: probe pulse and read-out pulse. Read-out amplitude $I_{\text {read }}$ is kept at a level just above the retrapping current $I_{r}$ for the recording of the switching events. Probe pulse amplitude $I_{p}$ is varied to probe the switching events. For a particular pulse amplitude, the number of switching events $(n)$ is counted, and the switching probability is defined as $P=n / N$. Current pulses with different polarities are used to further check the consistency of the measurement method. The time interval between two current pulses is set to $\tau_{3}=10 \mathrm{~ms}$ in order to ensure cooling of the electrons after the $\mathrm{JJ}$ retraps to the superconducting state.

Figure 3(b) shows the switching probability against the probe pulse amplitude at different bath temperatures for a sample with Au acting as normal metal. The probe pulse width $\tau_{1}$ and the read-out pulse width $\tau_{2}$ are set to $2 \mu$ s and $1 \mathrm{~ms}$. In our experiment, the shortest probe pulse width is limited by the bandwidth of the biasing circuit. With optimized design, one can reach nanosecond speed with this measurement technique. ${ }^{9}$ On the other hand, we find that the temperature resolution enhanced by increasing the probe pulse width and $I_{50}$ of the thermometer (see supplementary material for the temperature resolution of the thermometer). Current pulses with different polarities are plotted as dots and squares separately, and the results overlap as expected. The switching probability increases from 0 to 1 when increasing the probe pulse amplitude $I_{p}$. For thermometer calibration, we define $I_{50}$ as that corresponding to the switching probability $P=0.5$. In Fig. 3(c), we plot the temperature dependence of $I_{50}$ for different probe pulse widths together with quasi-DC sweeps of the bias current. For a fixed bath temperature, the longer pulse width gives higher switching probability of the JJ. Thus, smaller $I_{p}$ is needed to drive JJ to the normal state ${ }^{31}$ so that the measured $I_{50}$ is lower. For a pulse width of $256 \mu \mathrm{s}, I_{50}$ is nearly equal to $I_{s w}$ obtained from the DC measurement.

Instead of using DC current to elevate the electron temperature in the metal film in the steady state, we heat the electrons in the metal film with a rectangular current pulse. As in the steady state measurement, pulses with different 
polarities are used to check that no heating current flows through the probing lines. Meanwhile, by changing the time interval $\left(t_{\text {delay }}\right)$ between the probe pulse and the heat pulse with one of them fixed, we can use this technique to monitor the electron temperature in the metal film, while pulse heating is used to create a nonequilibrium on it. In Fig. 4(a), we show the electron temperature in the heated $\mathrm{Ag}$ films in response to a heat pulse applied at various bath temperatures. When the heating amplitude is increased from zero, electron temperature starts to rise and finally reaches the steady state by dissipating the Joule heat through e-p scattering as in steady state experiments. When heating is switched off, electrons start to relax and reach the bath temperature of the refrigerator again. For heat current pulses with inverted polarities, the electron temperatures show identical response as expected.

Figures 4(b) and 4(c) show the zoom-in of black and red squares of (a) for the bath temperature of $41 \mathrm{mK}$. In Fig. 4(b), one can see that electron temperature shows an almost linear dependence in time within the first few microseconds of the heating pulse. This can be explained by the weak e-p scattering when the temperature difference between electrons and phonons is small. In this case, almost all heating applied heats up the electrons in the metal film. Electron temperature changes in the metal film can then be written as $\Delta T_{e}=\frac{P_{J} \Delta t}{C_{e}}$. Here, $P_{J}$ is the Joule heating power, $C_{e}$ is the heat capacity of the metal film, and $\Delta t$ is the time delay between heating and probing pulses. As electron temperature increases further, the contribution of e-p scattering starts to dominate and the electron temperature shows a deviation from the linear dependence in time.

When switching off heating, electrons in metal films start to cool by e-p scattering. For this relaxation process, shown in Fig. 4(c), one can write down the thermal equation

$$
C_{e} \frac{d \Delta T_{e}}{d t}=-G_{t h} \Delta T_{e} .
$$

From Eq. (3), one finds $\Delta T_{e}=\Delta T_{e}(0) e^{-t / \tau}$. The thermal relaxation time is given by $\tau=\frac{C_{e}}{G_{t h}}$. Here, we assume $T_{p}=T_{e}$

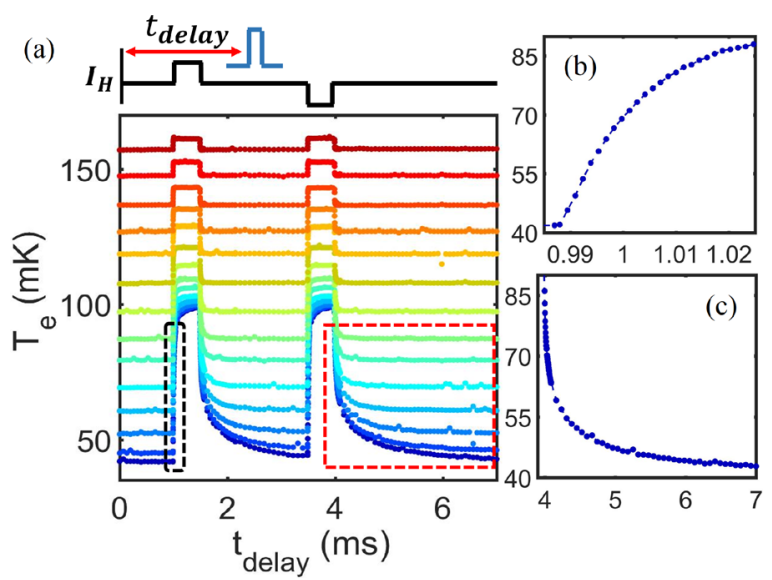

FIG. 4. (a) Electron temperature of metal films in response to a heating pulse with the bath temperature varying from 41 to $157 \mathrm{mK}$. $t_{\text {delay }}$ is defined as the time interval between the heat pulse (black) and the probe pulse (blue). Probe pulse width $\tau_{1}$ and read-out pulse width $\tau_{2}$ are set to $2 \mu \mathrm{s}$ and $1 \mathrm{~ms}$, respectively. (b) and (c) Are zoom-in of black and red squares (a) with the bath temperature of $41 \mathrm{mK}$. and that $\Delta T_{e}(0)$ is small enough so that $C_{e}$ and $G_{t h}$ can be approximated by their equilibrium values. As shown in Fig. 4(a), the relaxation process is faster at higher bath temperatures. With the technique shown above, one can monitor the electron temperature of metal films in a time-dependent nonequilibrium state. The measurement presented here can be used to determine the heat capacity and thermal relaxation time of normal metal films at sub-kelvin temperatures. The material-specific measurement results will be reported elsewhere.

The measurement technique for the nonequilibrium experiment with normal metal applies also to other systems once a well-defined Fermi distribution is formed during the measurement. In our experiment, the dimensions of the metal film are sufficiently small and simulation shows that the whole metal film has a uniform electron temperature while heating is applied. For a system with larger dimensions or with low electron density, a temperature gradient may exist within the system. The measured electron temperature then depends on where the thermometer is located in the system. To determine $I_{50}$ for each temperature, we need to send a pulse train shown in Fig. 3(a) repeatedly at each probe pulse amplitude and then scan the pulse amplitude. Also, the JJ needs to be initialized to zero resistance state after switching. Thus, the thermometer cannot be used in a continuous manner to track the system temperature. For a single-shot event, the measurement speed can reach the nanosecond range. ${ }^{9}$ With normal metal between two superconductors functioning as an absorber, one can use this technique in calorimetry. One may reduce the volume of the normal metal to about 0.1 $\mu \mathrm{m}^{3}$. At $50 \mathrm{mK}$, the predicted electronic heat capacity is $3 \times 10^{21} \mathrm{~J} / \mathrm{K}$. Incoming photons with a frequency of $20 \mathrm{GHz}$ will then cause a $4 \mathrm{mK}$ temperature rise out of $50 \mathrm{mK}$ equilibrium temperature, which is detectable using a JJ thermometer within the thermal relaxation time (see supplementary material for the temperature resolution of the thermometer). The thermal relaxation time is typically tens of microseconds at low temperature. ${ }^{32}$

In conclusion, we have demonstrated the fast thermometry based on proximity Josephson junctions. In a steady state measurement, we determined the heat transport via e-p coupling in Ag films. By employing rectangular pulses for heating and probing, we can monitor the electron temperature in metal films in time-dependent nonequilibrium. The measurement technique presented here can be used to explore phenomena in mesoscopic thermodynamics.

See supplementary material for the temperature resolution of the thermometer as a function of probe pulse width and $I_{50}$.

We acknowledge M. Meschke and J. T. Peltonen for technical help and D. Golubev for useful discussions. This work was supported by the Academy of Finland Center of Excellence program (Project Nos. 284594 and 273827). O.P.S. was supported by the Kavli Prize Postdoctoral Fellowship in Nanoscience and a grant from the Nokia Foundation Jorma Ollila Fund. We acknowledge the Micronova Nanofabrication Centre of Aalto University for providing the processing facilities. 
${ }^{1}$ K. Schwab, E. A. Henriksen, J. M. Worlock, and M. L. Roukes, Nature (London) 404, 974 (2000).

${ }^{2}$ M. Meschke, W. Guichard, and J. P. Pekola, Nature (London) 444, 187 (2006).

${ }^{3}$ A. Bérut, A. Arakelyan, A. Petrosyan, S. Ciliberto, R. Dillenschneider, and E. Lutz, Nature (London) 483, 187 (2012).

${ }^{4}$ D. R. Schmidt, C. S. Yung, and A. N. Cleland, Appl. Phys. Lett. 83, 1002 (2003).

${ }^{5}$ D. R. Schmidt, K. W. Lehnert, A. M. Clark, W. D. Duncan, K. D. Irwin, N. Miller, and J. N. Ullom, Appl. Phys. Lett. 86, 053505 (2005).

${ }^{6}$ S. Gasparinetti, K. L. Viisanen, O.-P. Saira, T. Faivre, M. Arzeo, M. Meschke, and J. P. Pekola, Phys. Rev. A 3, 014007 (2015).

${ }^{7}$ O.-P. Saira, M. Zgirski, K. L. Viisanen, D. S. Golubev, and J. P. Pekola, Phys. Rev. A 6, 024005 (2016).

${ }^{8}$ J. Govenius, R. E. Lake, K. Y. Tan, and M. Möttönen, Phys. Rev. Lett. 117, 030802 (2016).

${ }^{9}$ M. Zgirski, M. Foltyn, A. Savin, M. Meschke, and J. P. Pekola, e-print arXiv: 1704.04762.

${ }^{10}$ J. Wei, P. Cadden-Zimansky, and V. Chandrasekhar, Appl. Phys. Lett. 92, 102502 (2008).

${ }^{11}$ L. Angers, F. Chiodi, G. Montambaux, M. Ferrier, S. Guéron, H. Bouchiat, and J. C. Cuevas, Phys. Rev. B 77, 165408 (2008).

${ }^{12}$ J. M. Martinis, M. H. Devoret, and J. Clarke, Phys. Rev. B 35, 4682 (1987).

${ }^{13}$ P. Dubos, H. Courtois, B. Pannetier, F. K. Wilhelm, A. D. Zaikin, and G. Schön, Phys. Rev. B 63, 064502 (2001).

${ }^{14}$ W. A. Little, Can. J. Phys. 37, 334 (1959).
${ }^{15}$ V. F. Gantmakher, Rep. Prog. Phys. 37, 317 (1974).

${ }^{16}$ F. C. Wellstood, C. Urbina, and J. Clarke, Phys. Rev. 49(9), 5942-5955 (1994).

${ }^{17}$ J. T. Peltonen, P. Virtanen, M. Meschke, J. V. Koski, T. T. Heikkilä, and J. P. Pekola, Phys. Rev. Lett. 105, 097004 (2010).

${ }^{18}$ M. L. Roukes, M. R. Freeman, R. S. Germain, R. C. Richardson, and M. B. Ketchen, Phys. Rev. Lett. 55, 422 (1985).

${ }^{19}$ J. Rammer and A. Schmid, Phys. Rev. B 34, 1352 (1986).

${ }^{20}$ A. Sergeev and V. Mitin, Phys. Rev. B 61, 6041 (2000).

${ }^{21}$ J. F. DiTusa, K. Lin, M. Park, M. S. Isaacson, and J. M. Parpia, Phys. Rev. Lett. 68, 1156 (1992).

${ }^{22}$ S. Y. Hsu, P. J. Sheng, and J. J. Lin, Phys. Rev. B 60, 3940 (1999).

${ }^{23}$ M. E. Gershenson, D. Gong, T. Sato, B. S. Karasik, and A. V. Sergeev, Appl. Phys. Lett. 79, 2049 (2001).

${ }^{24}$ J. T. Karvonen, L. J. Taskinen, and I. J. Maasilta, Phys. Rev. B 72, 012302 (2005).

${ }^{25}$ A. Vinante, P. Falferi, R. Mezzena, and M. Mück, Phys. Rev. B 75, 104303 (2007).

${ }^{26}$ P. M. Echternach, M. T. Thoman, C. M. Gould, and H. M. Bozler, Phys. Rev. B 46, 10339 (1992).

${ }^{27}$ J. T. Karvonen and I. J. Maasilta, Phys. Rev. Lett. 99, 145503 (2007).

${ }^{28}$ S. Cojocaru and D. V. Anghel, Phys. Rev. B 93, 115405 (2016).

${ }^{29}$ J. M. Underwood, P. J. Lowell, G. C. O'Neil, and J. N. Ullom, Phys. Rev. Lett. 107, 255504 (2011).

${ }^{30}$ A. H. Steinbach and J. M. Martinis, Phys. Rev. Lett. 76, 3806 (1996).

${ }^{31}$ M. Foltyn and M. Zgirski, Phys. Rev. A 4, 024002 (2015).

${ }^{32}$ K. L. Viisanen and J. P. Pekola, e-print arXiv:1606.02985v1. 